\title{
Sex Differences in the Pharmacokinetics of Low-dose Ketamine in Plasma and Brain of Male and Female Rats
}

\author{
Samantha K. Saland and Mohamed Kabbaj \\ Department of Biomedical Sciences, Program in Neuroscience, College of Medicine, Florida State University, Tallahassee, \\ Florida
}

Received June 23, 2018; accepted September 10, 2018

\begin{abstract}
Recent work from our group and others has revealed a higher sensitivity of female rodents to the antidepressant-like effects of the $N$-methyl D-aspartate receptor antagonist ketamine strongly influenced by circulating estrogen and progesterone levels. However, in the absence of any preclinical studies of pharmacokinetic sex differences using low-dose ketamine in rats, it is unclear whether the effects of sex and hormonal milieu on ketamine's behavioral actions are influenced by differences in ketamine metabolism between male and female rats. Therefore, this work examined whether sex and hormonal status affect ketamine metabolism and distribution in male and female rats using a low antidepressant-like dose selectively effective in females. Intact male rats and female rats in either diestrus (low estrogen, progesterone) or proestrus (high estrogen,
\end{abstract}

progesterone) were administered low-dose ketamine, and their plasma and brains were collected to analyze levels of ketamine and its metabolites norketamine (NK) and dehydronorketamine. Females exhibited greater concentrations of ketamine and NK over the first 30 min following treatment in both brain and plasma, largely accounted for by slower clearance rates and longer halflives. Interestingly, despite the impact of ovarian hormones on behavioral sensitivity to ketamine, no appreciable differences in pharmacokinetic parameters existed between proestrus and diestrus female rats. This work is the first to demonstrate sex differences in ketamine pharmacokinetics in rats, and suggests that while sex differences in metabolism may influence the amount of ketamine and NK reaching target areas in the brain, the impact of circulating hormone levels here is negligible.

\section{Introduction}

The pressing need for more effective and faster-acting treatments for depression is underscored by the increasing global burden of depression as a leading cause of disability worldwide (Patel et al., 2016), owed in part to ignorance of complex disease mechanisms and stagnant progress in novel pharmacotherapeutic development. In light of this urgency, renewed hope was recently generated by the discovery that the noncompetitive $N$-methyl D-aspartate receptor antagonist ketamine can rapidly relieve depressive symptoms and suicidal ideation in many patients-notably among those with treatment-resistant depression (Abdallah et al., 2016). These findings have since been corroborated numerous times in clinical and preclinical settings, spurring significant efforts into understanding ketamine's underlying mechanisms with the goal of identifying new targets for rapid-acting treatments with sustained efficacy in a broader range of patients (Abdallah et al., 2016).

In the era of personalized medicine, greater emphasis on identifying biomarkers or predictors of rapid antidepressant response to ketamine has emerged in an attempt to address the large heterogeneity observed in patient response to currently available treatments (Zarate et al., 2013). Yet, despite

This work was supported by the National Institute of Mental Health [Grants MHR01 MH87583, MH099085].

https://doi.org/10.1124/jpet.118.251652. a well-established female preponderance in depressive disorders (Patel et al., 2016) and variable sex differences in antidepressant response (Keers and Aitchison, 2010), sex is a variable yet to be thoroughly investigated as a potential moderator of response to ketamine. Akin to genetic and environmental factors, sex is a naturally occurring disease and treatment modifier of particular interest (Keers and Aitchison, 2010; Becker et al., 2016), in that protective or treatment-enhancing factors in one sex may reveal prevention or treatment strategies in the other (de Vries and Forger, 2015).

Importantly, sex is a variable that influences nearly all pharmacokinetic processes-absorption, distribution, metabolism, and elimination-which might ultimately influence treatment response (de Vries and Forger, 2015). As a weak and highly lipophilic base, ketamine rapidly distributes to the brain upon administration primarily via passive diffusion across the blood-brain barrier. This parent drug is predominantly $N$-demethylated into norketamine (NK) and further transformed into dehydronorketamine (DHNK) and six diastereomeric hydroxynorketamine (HNK) metabolites (Mion and Villevieille, 2013)—all of which are blood-brain barrierpenetrant and pharmacologically active within the brain. On a basic level, the therapeutic efficacy and tolerability of ketamine is limited by the availability of the drug and/or its active metabolites in unbound form at relevant target sites within the brain and periphery, making pharmacokinetic

ABBREVIATIONS: AUC, area under the curve; DHNK, dehydronorketamine; HNK, hydroxynorketamine; HPC, hippocampus; IS, internal standard; LC, liquid chromatography; mPFC, medial prefrontal cortex; NK, norketamine; SPE, solid phase extraction. 
processes fundamental to the understanding of variability in treatment response across individuals both within and between sexes (Saland et al., 2017). Unfortunately, the lack of studies investigating sex differences in ketamine pharmacokinetics has left very little evidence regarding such effects in humans. The few that do exist suggest that observations of sex effects in ketamine metabolism and clearance are likely dose dependent (Sigtermans et al., 2009; Zarate et al., 2012).

As drug/metabolite levels available at the site(s) of action within the brain do not necessarily correlate with those in the periphery, preclinical studies can provide essential information not able to be directly obtained in humans. Indeed, recent work found HNK, but not ketamine or NK, levels to be greater in the brain of female mice following acute systemic administration of $10 \mathrm{mg} / \mathrm{kg}$ ketamine (intraperitoneally), in addition to greater female behavioral sensitivity to ketamine's antidepressant-like effects compared with males (Zanos et al., 2016). However, as in humans, circulating hormone levels were not controlled for in this study. It is clear that species- and dose-specific differences impede a clear understanding of how sex may influence ketamine pharmacokinetics and treatment response at low doses used in clinic.

Notably absent are related studies in rats, which may help bridge this gap in knowledge to paint a clearer picture of whether and how sex differences observed preclinically may apply to the human condition. As well, there is an absence of any information regarding how fluctuating hormone levels may influence ketamine pharmacokinetics, as they are known to affect antidepressant-like behavioral responses to this drug at the preclinical level (Carrier and Kabbaj, 2013; Saland et al., 2016). Therefore, herein we investigated ketamine, NK, and DHNK exposure levels in the plasma and brain of proestrus (high hormone) and diestrus (low hormone) female versus male rats following $2.5 \mathrm{mg} / \mathrm{kg}$ ketamine-a dose behaviorally effective in females but not in males. In addition, potential regional differences in the medial prefrontal cortex (mPFC) and hippocampus, depression- and ketamine-relevant brain regions, were examined independently.

\section{Methods}

\section{Animals}

Adult male (250-270 g) and female (200-225 g) Sprague-Dawley rats (Charles River, Wilmington, MA) were pair-housed in $43 \times 21.5 \times 25.5 \mathrm{~cm}$ plastic cages. Animals were maintained on a 12 hour: 12 hour light:dark cycle (lights on at 0700) in a temperature- and humidity-controlled room, and food and water were available ad libitum throughout the duration of the study. All animal protocols were carried out in accordance with the NIH Guide for Care and Use of Laboratory Animals and approved by the Institutional Animal Care and Use Committee of Florida State University.

\section{Estrous Cycle Monitoring}

Animals were habituated to handling procedures daily for 1 week upon arrival. Following habituation, daily estrous cycle monitoring and stage assignment of intact female rats was performed via vaginal lavage and characterization of cytologic smears as previously detailed (Hollis et al., 2011; Saland et al., 2016). Only rats exhibiting at least two consecutive 4-day cycles were used in the present work. Four female rats not meeting this criterion were excluded prior to group assignment and analysis. During this time, male rats received a similar brief daily handling treatment to minimize potential for stress and handling confounds between sexes.

\section{Pharmacokinetics Experimental Procedures}

Ketamine Treatment and Sample Collection. The complete experimental design and sample preparation workflow are illustrated in Fig. 1. Separate adult male rats ( $n=4 /$ time point) and female rats in either diestrus (low estrogen, progesterone, $n=4$ /time point) or proestrus (high estrogen, progesterone, $n=4$ /time point) received a single intraperitoneal (intraperitoneal) injection of ketamine hydrochloride (Butler Schein Animal Health, Inc., Dublin, $\mathrm{OH}$ ) at $2.5 \mathrm{mg} / \mathrm{kg}$, and were euthanized under nonstressful conditions after 5, 10, 30, 60, 90, or 180 min. Brains were immediately removed, snap-frozen in 2-methylbutane, and stored at $-80^{\circ} \mathrm{C}$, along with plasma separated from trunk blood, until further processing. Drug was administered at a volume of $1 \mathrm{ml} / \mathrm{kg}$. Ketamine was administered between 1400 and 1500, at an approximate time period when circulating levels of estradiol and progesterone have been shown to be elevated in proestrus and low in diestrus female rats (Becker et al., 2005). In accordance with our previous work (Carrier and Kabbaj, 2013; Saland et al., 2016), this time period was chosen to determine whether the relative levels of these hormones in females at the time of ketamine administration would influence its metabolism and/or distribution, in addition to analysis of sex effects on these parameters.

Sample Preparation. Two hundred sixty microliters of plasma was transferred to a prechilled $1.5 \mathrm{ml}$ microcentrifuge tube and centrifuged for $15 \mathrm{~min}$ at $2000 \mathrm{~g}$ at $4^{\circ} \mathrm{C}$ to pellet any contaminants. Supernatant was transferred to a clean tube and an appropriate volume of ketamine-d4 (Cerilliant, Round Rock, TX) was added as an internal standard (IS) at $100 \mathrm{ng} / \mathrm{ml}$ for a $300 \mu \mathrm{l}$ final volume. Samples were pulse-vortexed for 1 minute and then mixed on a shaker plate for $10 \mathrm{~min}$ at room temperature to equilibrate the IS. Spiked plasma $(250 \mu \mathrm{l})$ was then transferred to a clean, prechilled tube, diluted 1:1 with acidified water $(0.4 \mathrm{~N} \mathrm{HCl})$, vortexed, and stored at $4^{\circ} \mathrm{C}$ overnight to disrupt plasma protein binding and precipitate proteins. The following day, samples were thawed on ice, vortexed, and centrifuged for $20 \mathrm{~min}$ at $8000 \mathrm{~g}$ at room temperature to clarify prior to solid phase extraction (SPE). For brain tissue samples, ketamine$\mathrm{d} 4$ was added at $300 \mathrm{ng} / \mathrm{g}$ to frozen tissue punches $(1.0 \mathrm{~mm})$ collected from $200 \mu \mathrm{m}$ sections of the dorsal hippocampus (HPC) and medial prefrontal cortex (mPFC). Spiked tissue samples were homogenized via sonication in $200 \mu \mathrm{l}$ MilliQ water. Eight hundred microliters of $100 \%$ methanol $(\mathrm{MeOH})$ was immediately added and samples were vortexed for 1 minute to mix. Following homogenization, samples were sonicated in an ice bath for $15 \mathrm{~min}$ and stored at $4^{\circ} \mathrm{C}$ overnight. The following day, samples were briefly vortexed and centrifuged for $10 \mathrm{~min}$ at $3000 \mathrm{~g}$ at $4^{\circ} \mathrm{C}$. Supernatant was transferred to a clean tube, dried via SpeedVac, and resuspended in $500 \mu \mathrm{l} 0.2 \mathrm{~N} \mathrm{HCl}$.

For SPE, acidified samples (250 $\mu$ l for plasma, $500 \mu$ l for brain) were loaded onto Oasis MCX (1 cc/30 mg) cartridges (Waters, Milford, MA) preconditioned with $1 \mathrm{ml} \mathrm{MeOH}$ and $1 \mathrm{ml}$ MilliQ water and allowed to elute via gravity. Columns were washed with $1 \mathrm{ml} 0.1 \mathrm{~N} \mathrm{HCl}$, followed by $1 \mathrm{ml} \mathrm{MeOH}$. Ketamine, NK, and DHNK analytes were then eluted twice with $500 \mu \mathrm{l} 5 \% \mathrm{NH}_{4} \mathrm{OH}$ in $\mathrm{MeOH}$ via gravity and dried via SpeedVac. Pellets were stored at $-20^{\circ} \mathrm{C}$ until further processing.

\section{Quantification of Ketamine and Metabolites in Biologic Matrices}

HPLC for Plasma Samples. Dried plasma extracts after SPE were redissolved in $0.5 \%$ acetonitrile aqueous solution with $0.1 \%$ formic acid (1:2 v:v plasma:solvent). Two microliters of the above solutions was loaded to the NanoAquity nanoLC system (Waters) for liquid chromatography (LC) separations with a single pump trapping fluidic configuration. A Symmetry C18 $5 \mu \mathrm{m} 180 \mu \mathrm{m} \times 20 \mathrm{~mm}$ trap column was followed by a HSS T3 $1.8 \mu \mathrm{m} 75 \mu \mathrm{m} \times 150 \mathrm{~mm}$ analytical column (both by Waters) in a vented configuration. Buffer A was aqueous solution with $0.1 \%$ formic acid; buffer B was acetonitrile with $0.1 \%$ formic acid. The LC gradient profile was as follows with a flow 


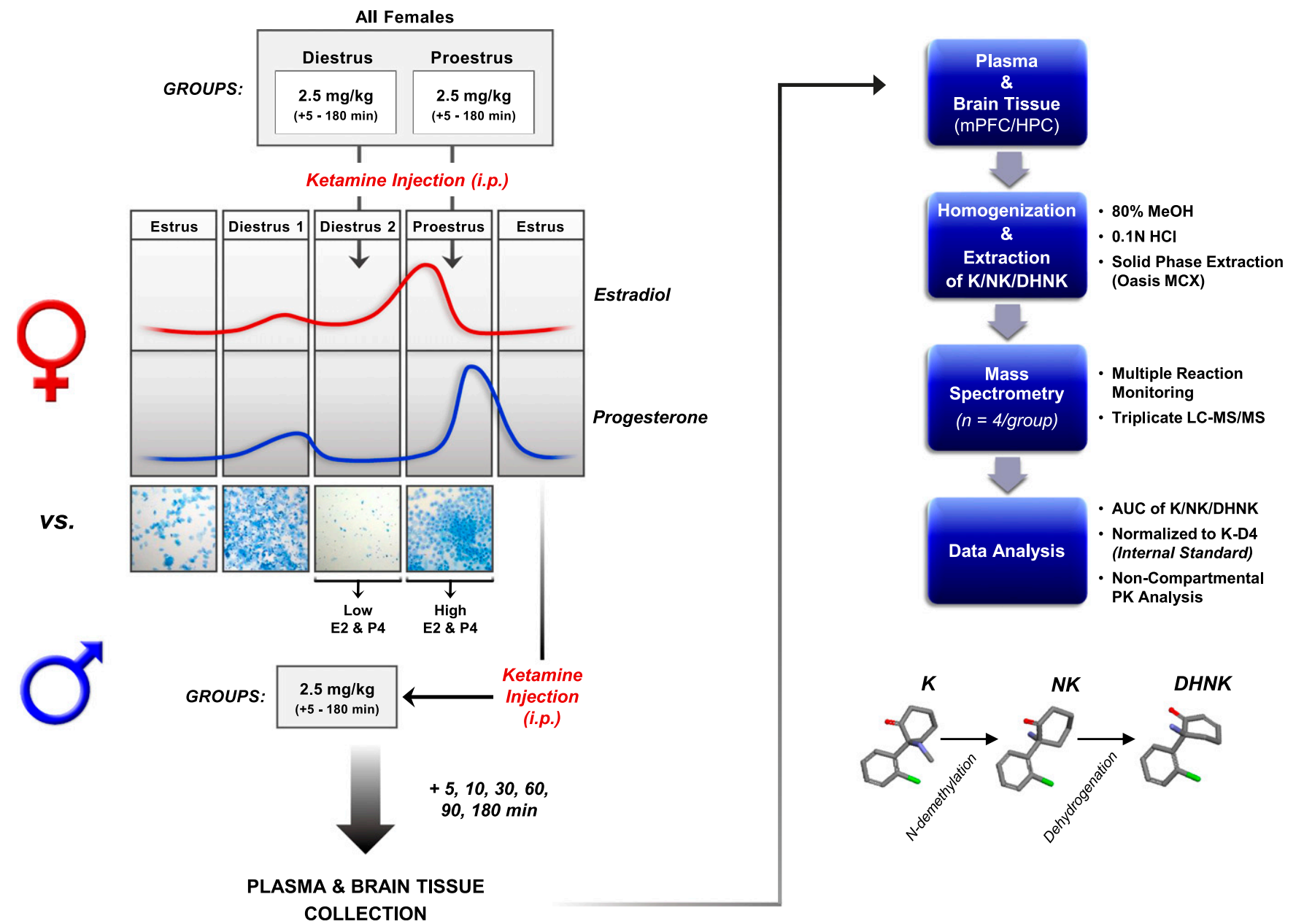

Fig. 1. Pharmacokinetics experimental design and sample preparation workflow. AUC, area under the curve; DHNK, dehydronorketamine; HCl, hydrochloric acid; HPC, hippocampus; K, ketamine; LC-MS/MS, liquid chromatography-tandem mass spectrometry; $\mathrm{MeOH}$, methanol; mPFC, medial prefrontal cortex; NK, norketamine; PK, pharmacokinetic.

rate of $400 \mathrm{nl} / \mathrm{min}: 1 \% \mathrm{~B}$ at $0 \mathrm{~min}, 85 \% \mathrm{~B}$ at $15-20 \mathrm{~min}$, and $1 \% \mathrm{~B}$ at 25-35 min.

HPLC for Brain Tissue Samples. Dried brain extracts after SPE were redissolved in $2 \%$ acetonitrile aqueous solution with $0.1 \%$ formic acid ( $1: 12 \mathrm{mg}: \mu \mathrm{l}$ tissue:solvent). Two microliters of the above solutions were loaded to the NanoAquity nanoLC system (Waters) for LC separations with a single pump trapping fluidic configuration. A Symmetry C18 $5 \mu \mathrm{m} 180 \mu \mathrm{m} \times 20 \mathrm{~mm}$ trap column was followed by a HSS T3 $1.8 \mu \mathrm{m} 150 \mu \mathrm{m} \times 100 \mathrm{~mm}$ analytical column (both by Waters) in a vented configuration. Buffer A was aqueous solution with $0.1 \%$ formic acid; buffer $\mathrm{B}$ was acetonitrile with $0.1 \%$ formic acid. The LC gradient profile was as follows with a flow rate of $2 \mu \mathrm{l} / \mathrm{min}$ : $1 \% \mathrm{~B}$ at $0 \mathrm{~min}, 85 \% \mathrm{~B}$ at $5-5.5 \mathrm{~min}$, and $1 \%$ $\mathrm{B}$ at $6-7 \mathrm{~min}$.

Mass Spectrometry. LC eluents were ionized in positive ion mode by nanoelectrospray ionization. Thus generated analyte ions were detected on-line with a Xevo TQ-S Triple Quadrupole Mass Spectrometer (Waters). We optimized the conditions for nanoelectrospray source as follows: $+3.3 \mathrm{kV}$ capillary voltage, $43 \mathrm{~V}$ cone voltage, $50 \mathrm{~V}$ source offset, $100^{\circ} \mathrm{C}$ source temperature, and 0.20 bar spray gas. Standard solutions were directly infused (without LC separation) to optimize the multiple reaction monitoring transitions for quantification: ketamine $(\mathrm{m} / z 238 \rightarrow 125)$, norketamine $(\mathrm{m} / z 224 \rightarrow 125)$, dehydronorketamine $(\mathrm{m} / z 222 \rightarrow 142)$, and ketamine-d4 (internal standard, IS, $m / z 242 \rightarrow 129$ ) with 3 -ms dwell time and $25 \mathrm{~V}$ collision energy. For plasma samples, seven calibration standards were prepared by spiking standards to "blank" plasma followed by SPE extraction. Concentration of those spiked standards was as follows: ketamine-d4 (IS) $100 \mathrm{ng} / \mathrm{ml}$; ketamine, norketamine, and dehydronorketamine $1,5,25,100,500,800$, and $1000 \mathrm{ng} / \mathrm{ml}$. The calibration linearity was observed for $1-500 \mathrm{ng} / \mathrm{ml}$ range. For brain tissue samples, five calibration standards were prepared by spiking standards to "blank" tissues followed by SPE extraction. Concentration of those spiked standards was as follows: ketamine-d4 (IS) $300 \mathrm{ng} / \mathrm{g}$; ketamine, norketamine, and dehydronorketamine 5, 25, 100, 500, and $1000 \mathrm{ng} / \mathrm{g}$. The calibration linearity was observed for $5-1000 \mathrm{ng} / \mathrm{g}$ range. Standard curves are presented in Fig. 2 for plasma (Fig. 2A) and brain tissue (Fig. 2B).

All samples and calibrants LC-MS/MS experiments were run in triplicates. TargetLynx software (Waters) was used to quantify automatically ketamine, norketamine, and dehydronorketamine in plasma and brain tissues. TargetLynx extracted the area under curve (AUC) of those targets of interest in the multiple reaction monitoring chromatograms with a restraint of retention time. TargetLynx then normalized the response by comparing the AUC to that of IS. Calibrants response was used to generate calibration curves that were used to calculate the concentration in the unknown samples.

\section{Pharmacokinetics Data Analysis}

Pharmacokinetic parameters following intraperitoneal ketamine administration in male and female rats were determined from mean plasma concentration-time data via noncompartmental analysis using 
A

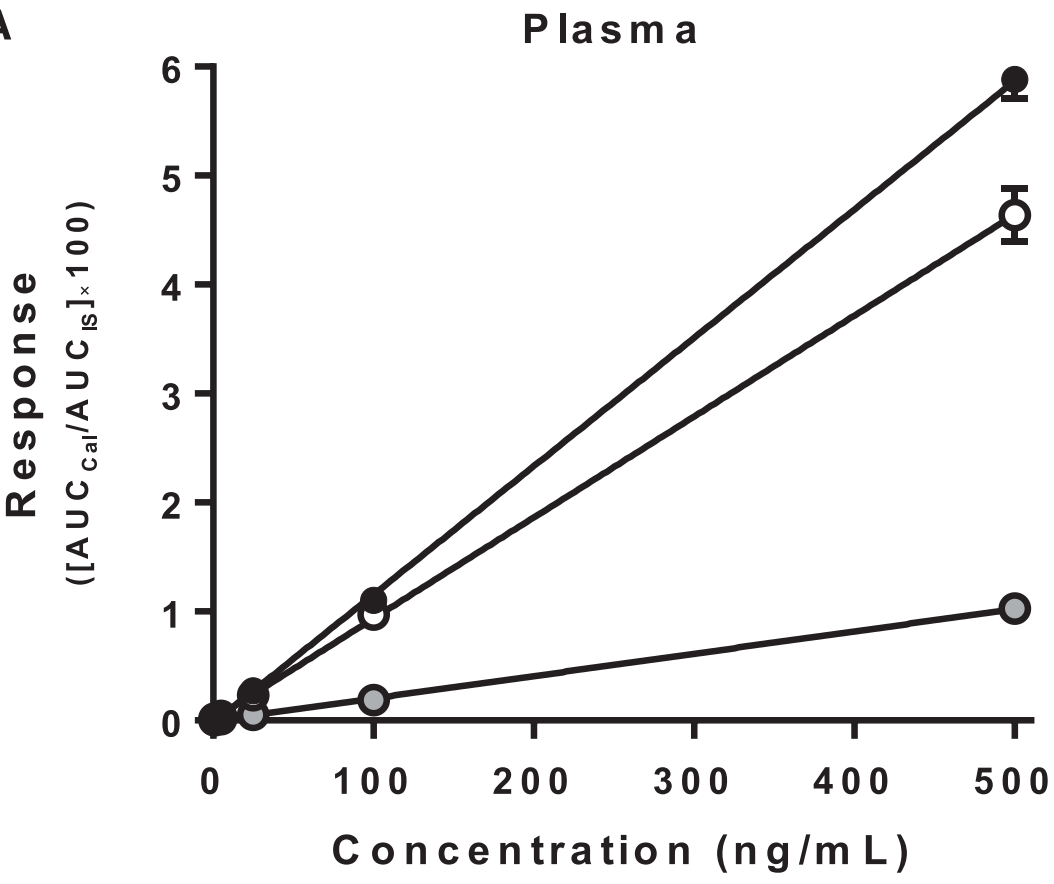
$\rightarrow \quad k$
$y=0.01178 x-0.02288$
$R^{2}=0.9998$

-O N K

$y=0.009266 x-0.01007$

$R^{2}=0.9999$

-O- DHNK

$y=0.002051 x-0.002223$

$R^{2}=0.9997$

B

\section{Brain}

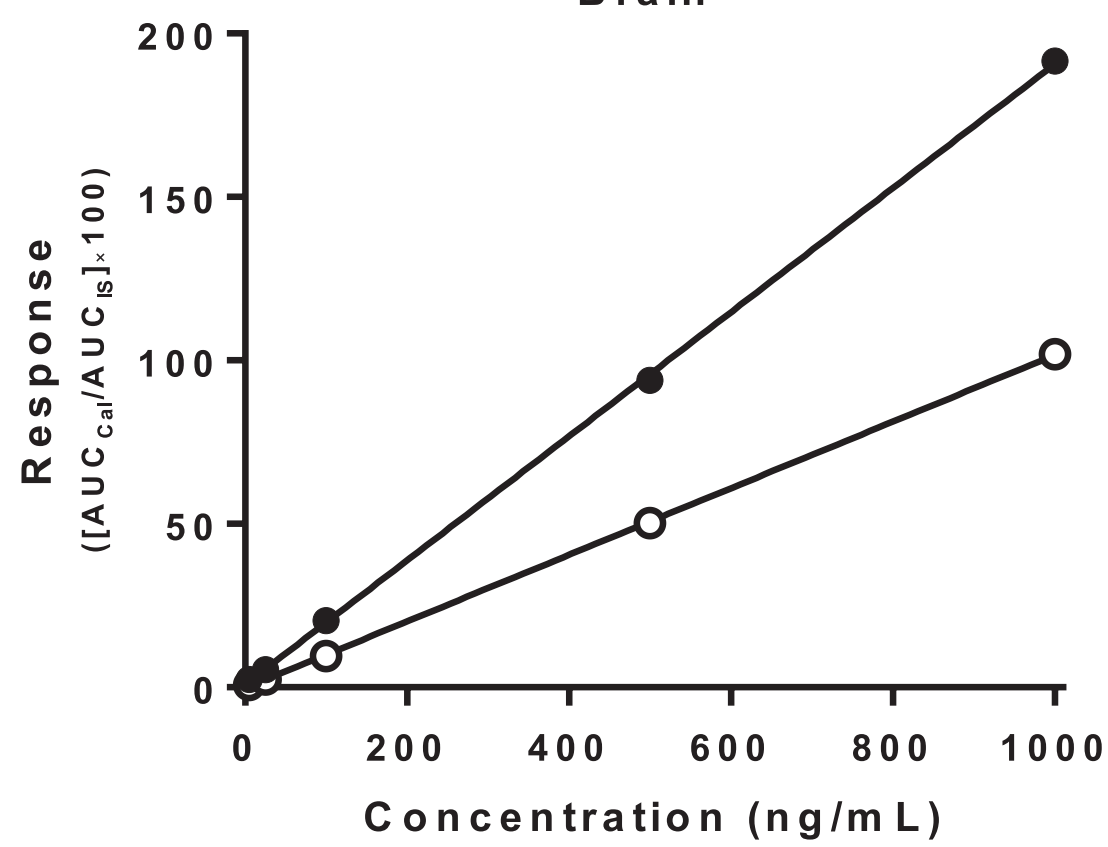


brain concentrations of ketamine and metabolites across time were analyzed by two-way analysis of variance, with sex/estrous cycle and time as independent factors. Due to the sparse sampling protocol required herein for parallel analysis of plasma and brain tissue, repeated-measures analysis across time points collected was not possible. Where appropriate, significant main or interaction effects were followed by Bonferroni's multiple comparisons test to determine between-group differences across time. Multiplicity-adjusted $P$ values are reported. GraphPad Prism 6.0 software (GraphPad Software Inc., San Diego, CA) was used for all statistical analyses and production of graphs. Alpha was set to 0.05 for all statistical analyses.

\section{Results}

\section{Plasma Concentrations of Ketamine and Metabolites in Male and Female Rats}

Following systemic administration of the same low dose of ketamine to male and female rats, plasma concentrations of the parent drug were significantly higher in female rats $5(P=$ $0.0367)$ and $10(P=0.0385)$ min posttreatment compared with male rats [Fig. 3A; sex: $F(1,59)=18.72, P<0.0001$; time: $F(5,59)=48.53, P<0.0001$; interaction: $F(5,59)=1.337$, $P=0.2615]$. Accordingly, greater NK levels in females versus
A
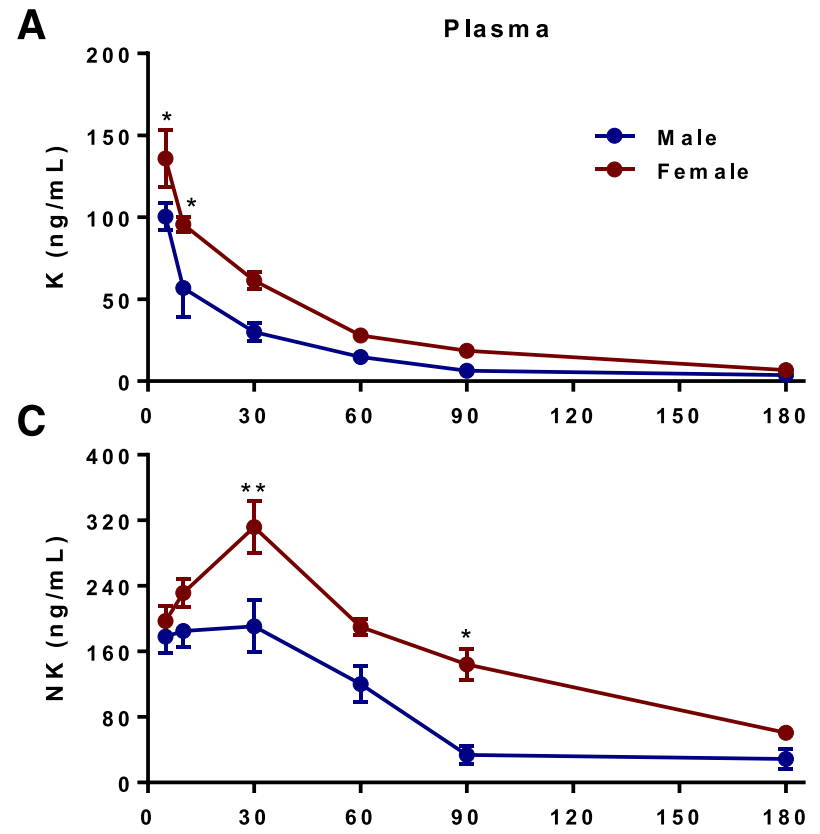

E

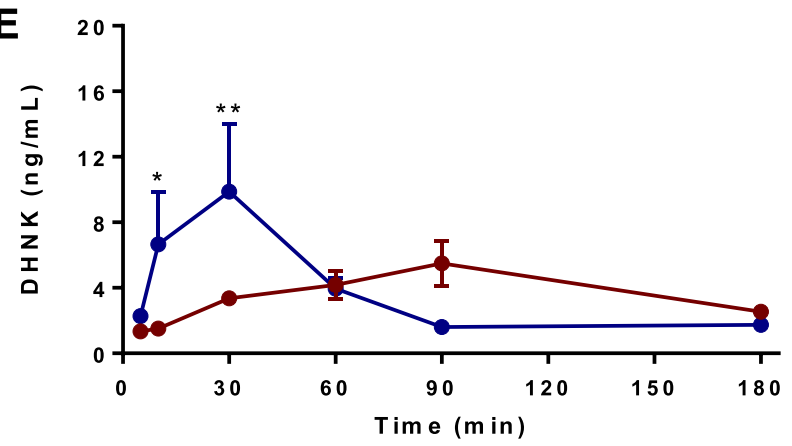

G

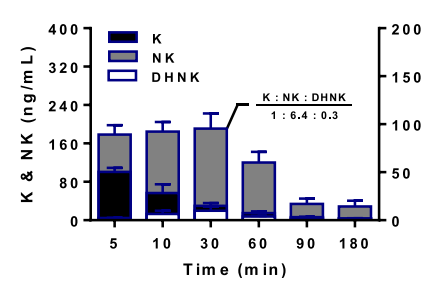

H

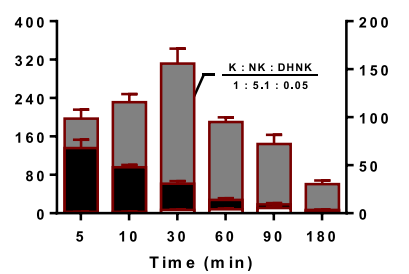

B
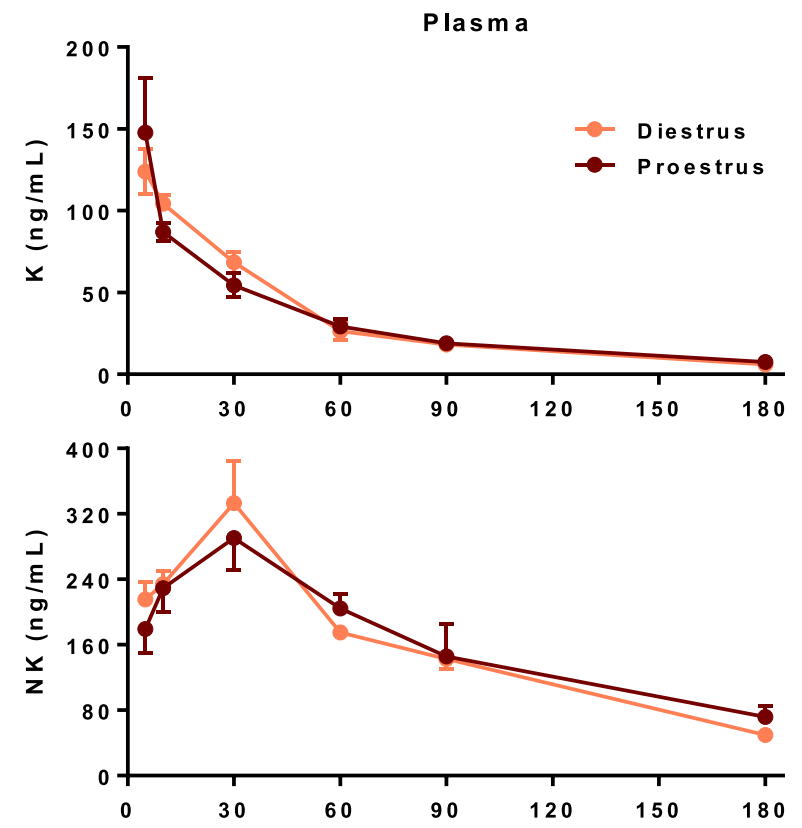

F

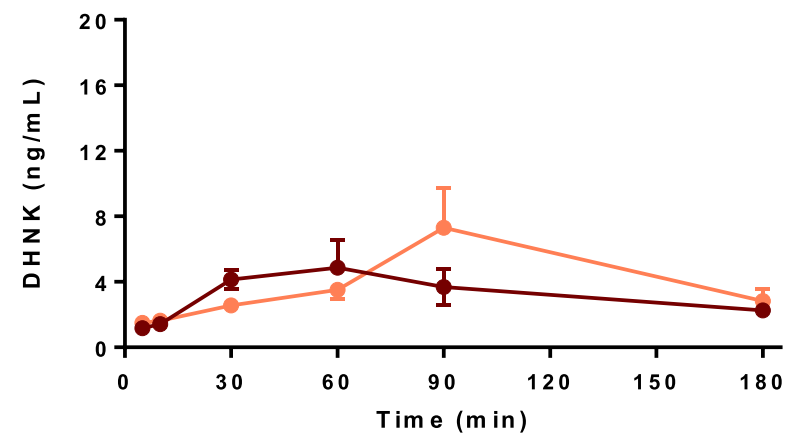

I

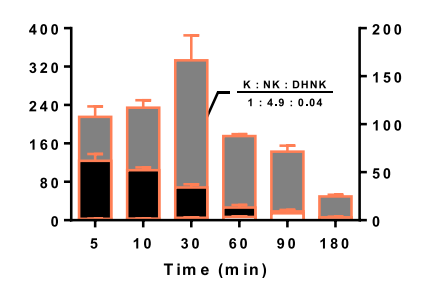

Fig. 3. Plasma concentration-time profiles of ketamine and its metabolites in male and cycling female rats. (a and b) Females displayed greater levels of ketamine $(\mathrm{K})$ rapidly upon intraperitoneal administration at $5(P=0.0367)$ and $10(P=0.0385)$ min postdose, relative to their male counterparts, regardless of hormonal status. (c and d) Higher concentrations of the norketamine (NK) metabolite were also subsequently observed in female rats at $30(P=0.0013)$ - and 90 -min $(P=0.0111)$ time points compared with males, in an estrous-cycle independent manner. (e) However, dehydronorketamine (DHNK) was significantly elevated in male compared with female rats $10(P=0.0328)$ and $30(P=0.0031)$ min after ketamine administration. (f) Estrous cycle had minimal influence on DHNK levels in females. (g-j) Superimposed K, NK, and DHNK concentrations reveal greater metabolite-to-parent concentrations in males than in females through the first 30 min posttreatment, despite their overall lower levels of $\mathrm{K}$ and $\mathrm{NK}$ during this time. $* P<$ 0.05 vs. male, ${ }^{* * P}<0.01$ vs. male. 
males were observed at subsequent $30(P=0.0013)$ - and 90-min $(P=0.0111)$ time points [Fig. 3C; sex: $F(1,57)=26.20, P<$ 0.0001 ; time: $F(5,57)=24.41, P<0.0001$; interaction: $F(5,57)=$ $1.708, P=0.1474]$. That DHNK levels were greater in male than female rats $10(P=0.0328)$ and $30(P=0.0031)$ min after ketamine administration suggests greater rate of transformation of DHNK from NK in males [Fig. 3E; sex: $F(1,60)=3.108$, $P=0.0830$; time: $F(5,60)=3.744, P=0.0051$; interaction: $F(5,60)=4.784, P=0.0010]$.

Interestingly, no meaningful differences were observed between diestrus and proestrus females in concentrations of ketamine [Fig. 3B; estrous cycle: $F(1,36)=0.003740$, $P=0.9516$; time: $F(5,36)=38.61, P<0.0001$; interaction: $F(5,36)=0.8209, P=0.5430]$, norketamine [Fig. 3D; estrous cycle: $F(1,36)=0.08903, P=0.7672$; time: $F(5,36)=18.65$, $P<0.0001$; interaction: $F(5,36)=0.5766, P=0.7175$ ], or dehydronorketamine [Fig. $3 \mathrm{~F}$; estrous cycle: $F(1,36)=0.2833$, $P=0.5978$; time: $F(5,36)=5.370, P=0.0009$; interaction: $F(5,36)=1.806, P=0.1365$ ], suggesting that ovarian hormones did not significantly influence ketamine pharmacokinetics at this low dose and route of administration during these cycle stages (see Tables 1 and 2 for pharmacokinetic parameter values).

\section{Brain Tissue Concentrations of Ketamine and Metabolites in Male and Female Rats}

We next sought to measure the active-site concentrations of ketamine and norketamine within key brain regions relevant to ketamine's antidepressant-like action. Ketamine levels in both the HPC (Fig. 4A) and mPFC (Fig. 4E) paralleled sex differences seen in plasma, with significantly greater concentrations of the parent drug in female rats 5 and $10 \mathrm{~min}$ posttreatment $(P$ values $<0.0001)$ relative to male rats $[\mathrm{HPC}$, sex: $F(1,59)=134.0, P<0.0001$; time: $F(5,59)=572.4, P<$ 0.0001 ; interaction: $F(5,59)=78.74, P<0.0001$; mPFC, sex:
$F(1,58)=6415, P<0.0001$; time: $F(5,58)=22557, P<0.0001$; interaction: $F(5,58)=3690, P<0.0001]$. Females also exhibited higher norketamine levels than males in both brain regions (Fig. 4, C and $\mathrm{G}$ ) rapidly beginning at $5 \mathrm{~min}$ up to 60 min after administration $(P$ values $<0.0001)$ [HPC, sex: $F(1,58)=179.4, P<0.0001$; time: $F(5,58)=112.1, P<0.0001$; interaction: $F(5,58)=12.94, P<0.0001$; mPFC, sex: $F(1,59)=$ 567.4, $P<0.0001$; time: $F(5,59)=251.8, P<0.0001$; interaction: $F(5,59)=42.59, P<0.0001]$. DHNK could not reliably be detected in all samples and was below the lower limit of quantitation at all time points in both brain regions and was, therefore, omitted from analysis.

As in plasma, while HPC NK was slightly greater in diestrus versus proestrus female rats, measured concentrations of ketamine and NK were markedly similar between these groups in the HPC [ketamine, Fig. 4B; estrous cycle: $F(1,35)=0.004494, P=0.9469$; time: $F(5,35)=468.2$, $P<0.0001$; interaction: $F(5,35)=0.1070, P=0.9900$; NK, Fig. 4D: estrous cycle: $F(1,34)=5.179, P=0.0293$; time: $F(5,34)=$ 113.6, $P<0.0001$; interaction: $F(5,34)=0.9861, P=0.4406]$ and $\mathrm{mPFC}$ [ketamine, Fig. $4 \mathrm{~F}$; estrous cycle: $F(1,36)=$ $0.07046, P=0.7992$; time: $F(5,36)=25491, P<0.0001$; interaction: $F(5,36)=1.326, P=0.2754$; NK, Fig. $4 \mathrm{H}$ : estrous cycle: $F(1,36)=1.179, P=0.2848$; time: $F(5,36)=277.4, P<$ 0.0001 ; interaction: $F(5,36)=0.2875, P=0.9168$ ]. The only exception was a modestly greater initial NK level in diestrus females $5 \mathrm{~min}(P=0.0413)$ postdose restricted to the HPC (Fig. 4D) relative to that of proestrus female rats (see Table 2 for pharmacokinetic parameter values).

\section{Sex Differences in Metabolism and Brain Distribution of Ketamine and Norketamine}

Concentration-time relationships between ketamine and its metabolites in plasma and brain tissues were examined to

TABLE 1

Pharmacokinetic parameters for ketamine and metabolites in plasma and brain tissue of male and female rats

\begin{tabular}{|c|c|c|c|c|c|c|c|c|c|c|c|c|}
\hline Parameter & $\mathrm{k}_{\mathrm{el}}$ & $t_{1 / 2}$ & $\mathrm{~T}_{\max }$ & $C_{\max }$ & $\mathrm{AUC}_{0-t}$ & $\mathrm{AUC}_{0-\infty}$ & $\mathrm{AUMC}_{0-t}$ & $\mathrm{AUMC}_{0-\infty}$ & $\mathrm{MRT}_{0-\mathrm{t}}$ & $\mathrm{MRT}_{0-\infty}$ & $\mathrm{V}_{\mathrm{z}} / \mathrm{F}$ & $\mathrm{Cl} / \mathrm{F}$ \\
\hline & $1 / \min$ & $\min$ & $\min$ & $n g / m l$ & $n g \bullet \min / l$ & $n g \bullet \min / l$ & $n g \bullet \min ^{2} / l$ & $n g \bullet \min ^{2} / l$ & $\min$ & $\min$ & $l / k g$ & $(l / \mathrm{min}) / \mathrm{kg}$ \\
\hline \multicolumn{13}{|l|}{ Plasma } \\
\hline \multicolumn{13}{|l|}{ Netamine } \\
\hline Male & 0.0156 & 44.42 & 5 & 100.52 & 2.86 & 3.10 & 127.52 & 186.09 & 44.60 & 60.05 & 51.69 & 0.807 \\
\hline Female & 0.0116 & 59.99 & 5 & 135.83 & 5.48 & 6.07 & 276.24 & 434.03 & 50.45 & 71.53 & 35.66 & 0.412 \\
\hline \multicolumn{13}{|c|}{ Norketamine } \\
\hline Male & 0.0124 & 55.76 & 30 & 190.68 & 14.54 & 16.85 & 808.15 & 1409.02 & 55.58 & 83.63 & 11.94 & 0.148 \\
\hline Female & 0.0095 & 72.72 & 30 & 311.80 & 28.04 & 34.41 & 1937.45 & 3751.16 & 69.10 & 109.03 & 7.62 & 0.073 \\
\hline \multicolumn{13}{|c|}{ Dehydronorketamine } \\
\hline Male & - & - & 30 & 9.88 & 0.61 & - & 38.48 & - & 62.67 & - & - & - \\
\hline \multirow{2}{*}{\multicolumn{13}{|c|}{$\mathrm{mPFC}$}} \\
\hline & & & & & & & & & & & & \\
\hline \multicolumn{13}{|l|}{ Ketamine } \\
\hline Male & 0.0471 & 14.72 & 5 & 198.47 & 9.09 & - & 288.26 & - & 31.72 & - & - & - \\
\hline Female & 0.0504 & 13.74 & 10 & 402.87 & 10.92 & - & 274.57 & - & 25.15 & - & - & - \\
\hline \multicolumn{13}{|c|}{ Norketamine } \\
\hline Male & 0.0223 & 31.08 & 10 & 170.64 & 9.78 & - & 405.59 & - & 41.45 & - & - & - \\
\hline Female & 0.0170 & 40.66 & 10 & 413.26 & 26.07 & - & 1233.84 & - & 47.33 & - & - & - \\
\hline \multicolumn{13}{|l|}{ HPC } \\
\hline \multicolumn{13}{|l|}{ Ketamine } \\
\hline Male & 0.0487 & 14.24 & 10 & 199.21 & 9.12 & - & 289.12 & - & 31.70 & - & - & - \\
\hline Female & 0.0503 & 13.78 & 10 & 375.79 & 10.72 & - & 277.72 & - & 25.91 & - & - & - \\
\hline \multicolumn{13}{|c|}{ Norketamine } \\
\hline Male & 0.0239 & 29.01 & 10 & 170.25 & 9.10 & - & 355.52 & - & 39.06 & - & - & - \\
\hline Female & 0.0163 & 42.42 & 10 & 374.46 & 21.69 & - & 1043.47 & - & 48.12 & - & - & - \\
\hline
\end{tabular}


TABLE 2

Pharmacokinetic parameters for ketamine and metabolites in plasma and brain tissue of diestrus and proestrus female rats

\begin{tabular}{|c|c|c|c|c|c|c|c|c|c|c|c|c|}
\hline Parameter & $\mathrm{k}_{\mathrm{el}}$ & $t_{1 / 2}$ & $\mathrm{~T}_{\max }$ & $C_{\max }$ & $\mathrm{AUC}_{0-\mathrm{t}}$ & $\mathrm{AUC}_{0-\infty}$ & $\mathrm{AUMC}_{0-t}$ & $\mathrm{AUMC}_{0-\infty}$ & $\mathrm{MRT}_{0-t}$ & $\mathrm{MRT}_{0-\infty}$ & $\mathrm{V}_{\mathrm{z}} / \mathrm{F}$ & $\mathrm{Cl} / \mathrm{F}$ \\
\hline & $1 / \min$ & $\min$ & $\min$ & $n g / m l$ & $n g \bullet \min / l$ & $n g \bullet \min / l$ & $n g \bullet \mathrm{min}^{2} / l$ & $n g \bullet \min ^{2} / l$ & $\min$ & $\min$ & $\mathrm{l} / \mathrm{kg}$ & $(l / \mathrm{min}) / \mathrm{kg}$ \\
\hline \multicolumn{13}{|l|}{ Plasma } \\
\hline \multicolumn{13}{|l|}{ Ketamine } \\
\hline Diestrus & 0.0122 & 56.70 & 5 & 123.93 & 5.56 & 6.06 & 270.64 & 400.96 & 48.64 & 66.15 & 33.74 & 0.412 \\
\hline Proestrus & 0.0110 & 63.15 & 5 & 147.73 & 5.38 & 6.07 & 281.36 & 468.94 & 52.33 & 77.27 & 37.53 & 0.412 \\
\hline \multicolumn{13}{|l|}{ Norketamine } \\
\hline Diestrus & 0.0108 & 64.21 & 30 & 333.05 & 27.39 & 31.99 & 1817.55 & 3070.51 & 66.36 & 96.00 & 7.24 & 0.078 \\
\hline Proestrus & 0.0085 & 81.22 & 30 & 290.54 & 28.63 & 37.03 & 2050.42 & 4548.97 & 71.62 & 122.83 & 7.91 & 0.068 \\
\hline \multicolumn{13}{|c|}{ Dehydronorketamine } \\
\hline Diestrus & - & - & 90 & 7.30 & 0.73 & - & 72.73 & - & 99.40 & - & - & - \\
\hline \multirow{2}{*}{\multicolumn{13}{|c|}{$\mathrm{mPFC}$}} \\
\hline & & & & & & & & & & & & \\
\hline \multicolumn{13}{|l|}{ Ketamine } \\
\hline Diestrus & 0.0505 & 13.74 & 10 & 404.82 & 10.91 & - & 274.31 & - & 25.14 & - & - & - \\
\hline Proestrus & 0.0504 & 13.74 & 10 & 400.93 & 10.92 & - & 274.83 & - & 25.17 & - & - & - \\
\hline \multicolumn{13}{|l|}{ Norketamine } \\
\hline Diestrus & 0.0167 & 41.57 & 10 & 415.86 & 26.49 & - & 1255.24 & - & 47.39 & - & - & - \\
\hline Proestrus & 0.0175 & 39.67 & 10 & 410.66 & 25.63 & - & 1210.16 & - & 47.21 & - & - & - \\
\hline \multicolumn{13}{|l|}{ HPC } \\
\hline \multicolumn{13}{|l|}{ Ketamine } \\
\hline Diestrus & 0.0500 & 13.87 & 5 & 373.87 & 10.69 & - & 276.91 & - & 25.90 & - & - & - \\
\hline Proestrus & 0.0504 & 13.76 & 10 & 380.44 & 10.74 & - & 278.37 & - & 25.92 & - & - & - \\
\hline \multicolumn{13}{|l|}{ Norketamine } \\
\hline Diestrus & 0.0163 & 42.47 & 10 & 383.14 & 22.99 & - & 1115.64 & - & 48.53 & - & - & - \\
\hline Proestrus & 0.0164 & 42.38 & 10 & 362.88 & 20.39 & - & 971.90 & - & 47.67 & - & - & - \\
\hline
\end{tabular}

,$- \mathrm{n} / \mathrm{a}$.

identify whether sex and/or hormonal milieu influence metabolism and central nervous system distribution of low-dose ketamine in behaviorally relevant brain regions. As DHNK was not reliably detected at or above quantifiable levels in brain tissue, primary emphasis was on ketamine and norketamine in the present analyses. To compare directly ketamine and metabolite exposure levels, their plasma (nanograms per milliliter) and brain tissue (nanograms per gram) concentrations-assuming a specific mass of $1 \mathrm{~g} / \mathrm{ml}$-were first converted into micromolars $(\mu \mathrm{M})$ to correct for molecular weight differences between molecules which contribute to overall AUC (exposure) values. The results of these comparisons are depicted graphically in Fig. 5 for visual comparison of exposure levels between sexes across time-numerical values presented therein (Fig. 5) are provided in Table 3, along with female-to-male ratios for comparison at each parameter.

\section{Discussion}

Comparative evaluation of pharmacokinetic profiles and brain tissue distribution of low-dose ketamine and its metabolites in male and female rats is necessary for improved understanding of their differential behavioral sensitivity to the drug observed preclinically and translation of such information across species. In this work, we provide the first characterization of ketamine pharmacokinetics across sex and hormonal status in rats following a low-dose administration of $2.5 \mathrm{mg} / \mathrm{kg}$ - a dose selectively effective in female but not male rats in eliciting antidepressant-like and prohedonic behaviors. Here, females exhibited greater peak ketamine and norketamine (NK) levels rapidly upon systemic administration in both plasma and two depression-relevant brain regions, the medial prefrontal cortex (mPFC) and hippocampus (HPC). Longer half-lives and slower clearance rates in females contributed to their greater exposure levels of ketamine and its primary metabolite over the 3-hour time course. Notably, while our previous work demonstrated an important role for ovarian hormones in the enhanced female behavioral sensitivity to low-dose ketamine (Carrier and Kabbaj, 2013; Saland et al., 2016), proestrus and diestrus female rats exhibited remarkably similar pharmacokinetic profiles, suggesting a more prominent influence of sex hormones on pharmacodynamic rather than pharmacokinetic systems in conferring sexdependent behavioral sensitivity to ketamine.

Absent from preclinical work are pharmacokinetic analyses of ketamine and metabolite concentrations across a broad range of doses within which behaviorally effective doses of ketamine reside. Therefore, it was of particular interest here to determine concentrations of ketamine and its metabolites for a low dose of ketamine that exerts antidepressant-like effects in female rats, but not in males, to obtain results that directly parallel behavioral and molecular findings presented in our previous studies utilizing the same low dose (Carrier and Kabbaj, 2013; Saland et al., 2016; Sarkar and Kabbaj, 2016). Following systemic administration of $2.5 \mathrm{mg} / \mathrm{kg}$ ketamine, ketamine concentrations peaked rapidly 5 min after dosing the plasma of male and female rats. Notably, peak concentrations of ketamine in female rats were significantly greater than those in males for the first $10 \mathrm{~min}$ after the drug was administered. Concentrations of its primary metabolite NK peaked slightly later at $30 \mathrm{~min}$ in both sexes as ketamine declined, exhibiting greater levels in female rats from its peak time through $90 \mathrm{~min}$ following treatment compared with males. Interestingly, DHNK (derived from NK) showed an opposite trend, in which males displayed greater and earlier peak concentrations at 30 min than females, whose levels peaked much later around $90 \mathrm{~min}$. Over the time points examined, AUC values indicated that total exposure levels to both ketamine and NK in females were nearly double those observed in males, whereas DHNK exposure was similar 

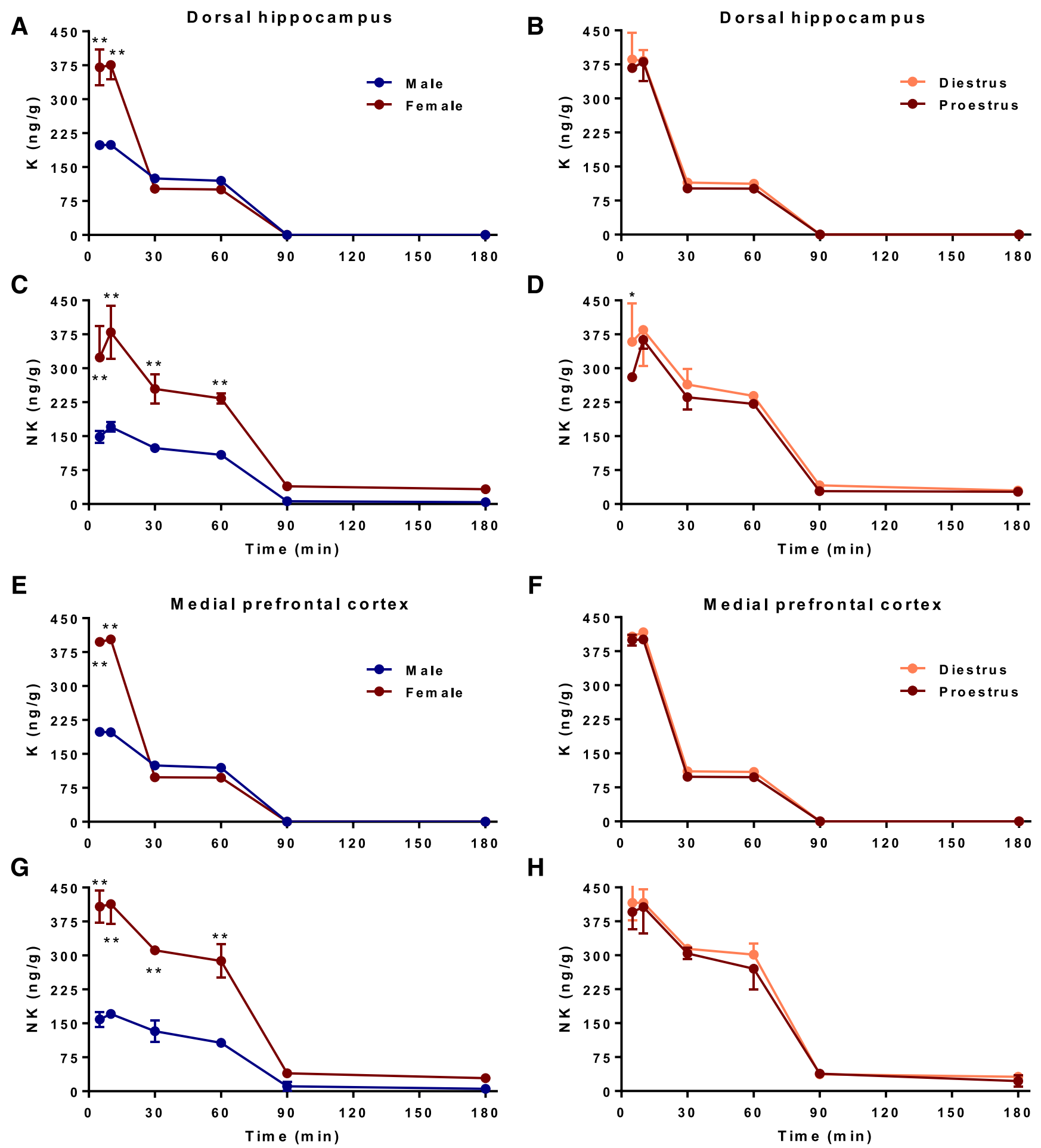

H

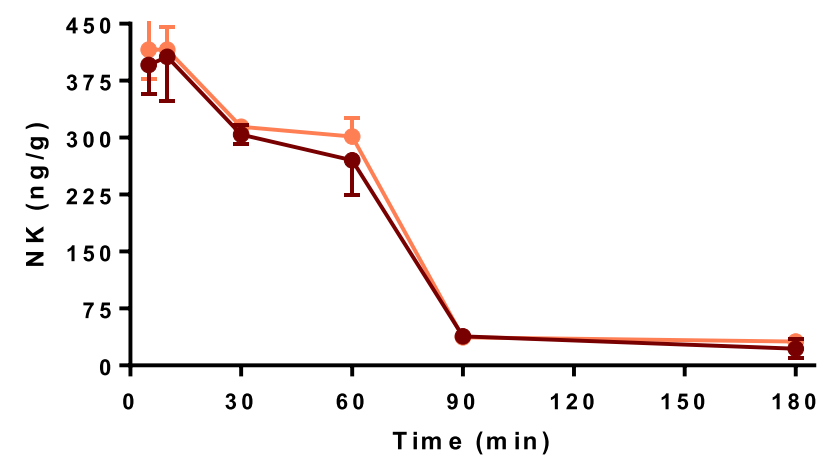

Fig. 4. Brain concentration-time profiles of ketamine and norketamine in male and cycling female rats. (a and e) Concentrations of ketamine (K) were $\sim 2$-fold greater in female compared with male rats $5-10 \min (P<0.0001)$ following administration in the hippocampus (HPC) and medial prefrontal cortex (mPFC), regardless of estrous cycle stage (b and f). (c and g) These differences were more pronounced for its metabolite norketamine (NK), whose levels in females were significantly higher 5-60 min posttreatment in both regions $(P<0.0001)$ compared with those in males. $(\mathrm{d}$ and $\mathrm{h})$ While NK levels in the HPC were higher in diestrus than in proestrus females 5 min after ketamine treatment $(P=0.0413)$, hormonal status had minimal effect on distribution of the parent drug and its metabolite within the HPC and mPFC. Data are expressed as mean \pm S.E.M. ( $n=3$ to $4 /$ group/time point). ** $P<$ 0.0001 vs. male, $* P<0.05$ vs. proestrus.

between sexes. Longer half-lives and slower clearance rates of both ketamine and NK in females contributed to their overall greater exposure levels compared with males. Given that the same trends in these parameters were observed for both ketamine and its primary metabolite NK, greater peak concentrations of ketamine in the plasma of females cannot be explained by more extensive or rapid metabolism to NK in males. Interestingly, male rats did exhibit a larger apparent 
A

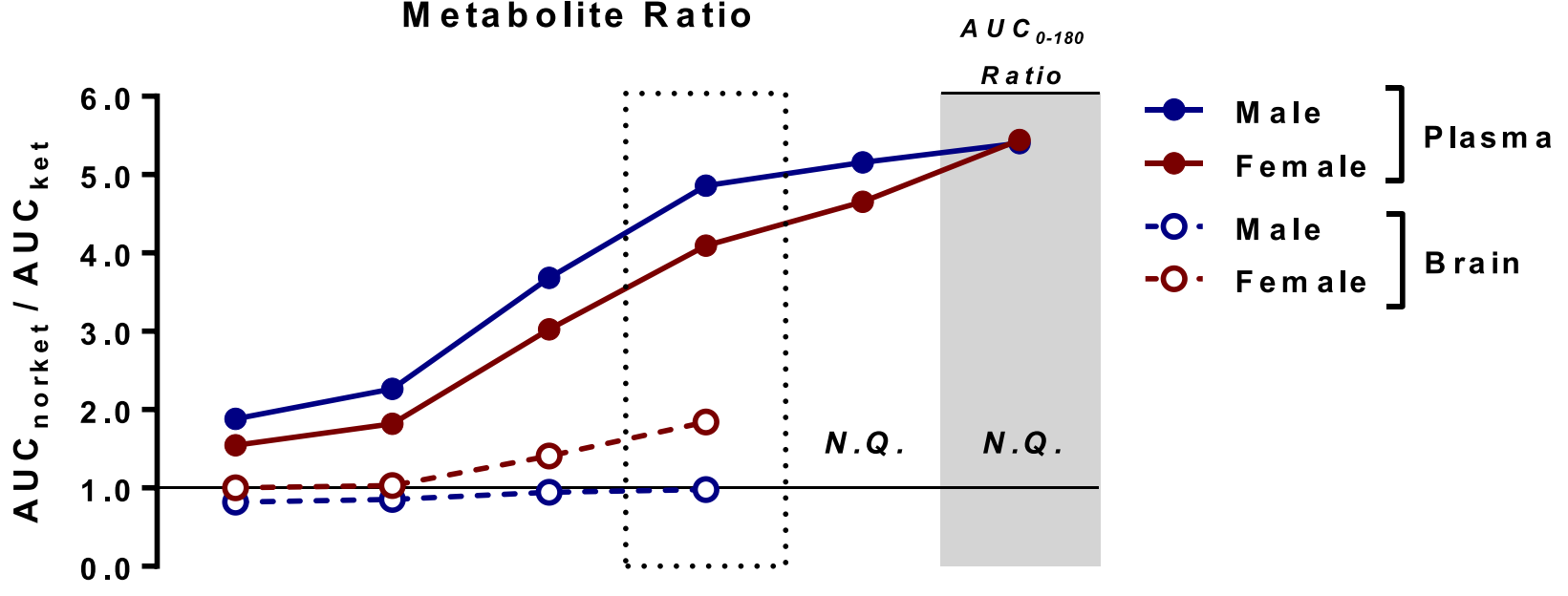

B

B rain-to-P las m a R atio

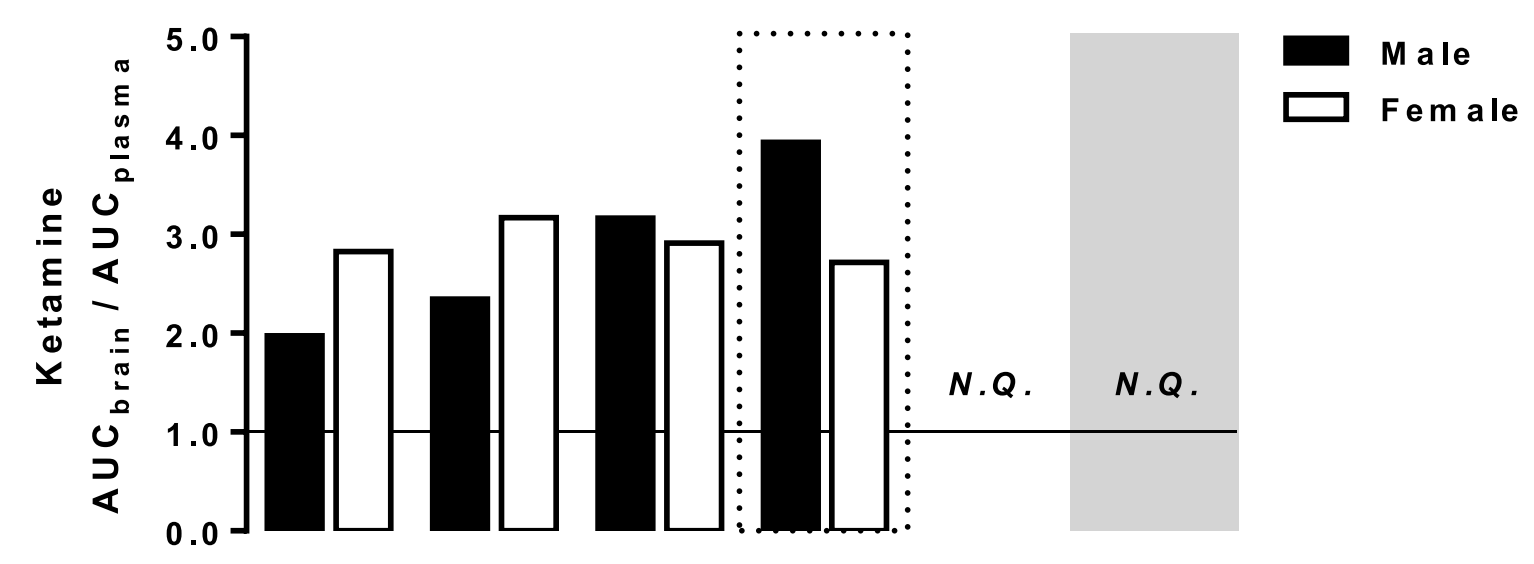

C

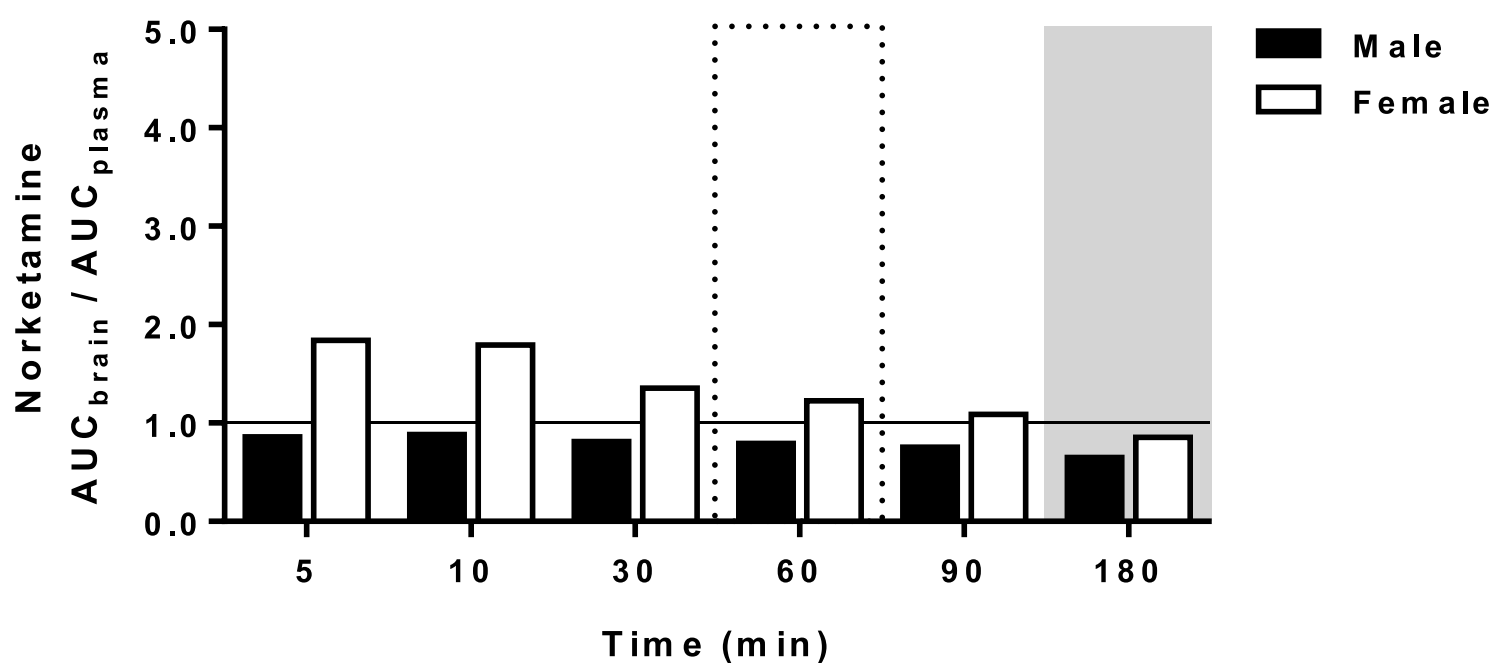

Fig. 5. Metabolite ratios and brain distribution of ketamine and norketamine in male and cycling female rats. (a) Ratio of norketamine to ketamine area under the concentration-time curve (AUC) values in plasma and brain of male ( $n=3$ to $4 /$ time point) and female ( $n=7$ to $8 /$ time point) rats (brain regions were averaged due to overall similarity). (b and c) Cumulative brain-to-plasma AUC ratios for ketamine and norketamine, respectively, over time in male and female rats. Data expressed as ratios of $\mathrm{AUC}_{0-t}$ values calculated using pooled group data from biologic replicate measurements at each time point_-plasma $(\mathrm{ng} / \mathrm{ml})$ and brain tissue $(\mathrm{ng} / \mathrm{g})$ concentrations were converted into micromolar $(\mu \mathrm{M})$ units beforehand for direct comparison between analytes. $\mathrm{AUC}_{0-180}$ ratio: total analyte exposure across all time points measured for each analysis, depicted by gray vertical bar. N.Q., not quantifiable, ketamine detected in brain samples < lower limit of quantitation $90-180 \mathrm{~min}$; here, $\mathrm{AUC}_{0-60}$ represents total exposure up to this time point, indicated by a dashed vertical bar for comparison. 
TABLE 3

Comparison of metabolite and brain-to-plasma ratios in male and female rats following low-dose ketamine administration

\begin{tabular}{|c|c|c|c|c|c|c|c|c|c|c|c|c|c|c|c|}
\hline \multirow{5}{*}{$\begin{array}{c}\text { Parameter } \\
\text { Minutes }\end{array}$} & \multicolumn{9}{|c|}{ Metabolite-to-Parent Ratios } & \multirow{2}{*}{\multicolumn{6}{|c|}{$\frac{\text { Brain-to-Plasma Ratios }}{\mathrm{AUC}_{\text {brain }} / \mathrm{AUC}_{\text {plasma }}}$}} \\
\hline & \multicolumn{3}{|c|}{$\mathrm{AUC}_{\mathrm{NK}} / \mathrm{AUC}_{\mathrm{K}}$} & \multicolumn{3}{|c|}{$\mathrm{AUC}_{\mathrm{DHNK}} / \mathrm{AUC}_{\mathrm{K}}$} & \multicolumn{3}{|c|}{$\mathrm{AUC}_{\mathrm{NK}}+\mathrm{DHNK} / \mathrm{AUC}_{\mathrm{K}}$} & & & & & & \\
\hline & \multicolumn{3}{|c|}{$\mu \mathrm{mol} \bullet \min / l$} & \multicolumn{3}{|c|}{$\mu \mathrm{mol} \bullet \min / l$} & \multicolumn{3}{|c|}{$\mu \mathrm{mol} \bullet \min / l$} & \multicolumn{6}{|c|}{$\mu \mathrm{mol} \bullet \min / l$} \\
\hline & \multirow[b]{2}{*}{ Male } & \multirow[b]{2}{*}{ Female } & \multirow[b]{2}{*}{$\mathrm{F}: \mathrm{M}$} & \multirow[b]{2}{*}{ Male } & \multirow[b]{2}{*}{ Female } & \multirow[b]{2}{*}{$\mathrm{F}: \mathrm{M}$} & \multirow[b]{2}{*}{ Male } & \multirow[b]{2}{*}{ Female } & \multirow[b]{2}{*}{$\mathrm{F}: \mathrm{M}$} & \multicolumn{3}{|c|}{ Ketamine } & \multicolumn{3}{|c|}{ Norketamine } \\
\hline & & & & & & & & & & Male & Female & $\mathrm{F}: \mathrm{M}$ & Male & Female & $\mathrm{F}: \mathrm{M}$ \\
\hline \multicolumn{16}{|l|}{ Plasma } \\
\hline 5 & 1.88 & 1.54 & -1.22 & 0.01 & 0.01 & -1.20 & 1.91 & 1.55 & -1.23 & - & - & - & - & - & - \\
\hline 10 & 2.27 & 1.82 & -1.24 & 0.04 & 0.01 & -3.47 & 2.31 & 1.84 & -1.26 & - & - & - & - & - & - \\
\hline 30 & 3.68 & 3.02 & -1.22 & 0.14 & 0.03 & -5.37 & 3.82 & 3.05 & -1.25 & - & - & - & - & - & - \\
\hline 60 & 4.86 & 4.09 & -1.19 & 0.19 & 0.05 & -3.93 & 5.06 & 4.14 & -1.22 & - & - & - & - & - & - \\
\hline 90 & 5.16 & 4.65 & -1.11 & 0.21 & 0.08 & -2.67 & 5.37 & 4.73 & -1.13 & - & - & - & - & - & - \\
\hline 180 & 5.40 & 5.44 & -0.99 & 0.23 & 0.13 & -1.78 & 5.64 & 5.57 & -1.01 & - & - & - & - & - & - \\
\hline \multicolumn{16}{|l|}{$\mathrm{mPFC}$} \\
\hline 5 & 0.85 & 1.09 & 1.28 & - & - & - & - & - & - & 1.97 & 2.93 & 1.48 & 0.89 & 2.07 & 2.32 \\
\hline 10 & 0.88 & 1.09 & 1.24 & - & - & - & - & - & - & 2.27 & 3.28 & 1.45 & 0.88 & 1.96 & 2.23 \\
\hline 30 & 0.94 & 1.49 & 1.59 & - & - & - & - & - & - & 3.15 & 2.97 & -1.06 & 0.80 & 1.47 & 1.82 \\
\hline 60 & 0.95 & 2.00 & 2.09 & - & - & - & - & - & - & 4.05 & 2.75 & -1.47 & 0.79 & 1.34 & 1.69 \\
\hline HPC & - & - & - & - & - & - & & & & & & & & & \\
\hline 5 & 0.80 & 0.91 & 1.15 & - & - & - & - & - & - & 1.97 & 2.73 & 1.38 & 0.83 & 1.62 & 1.94 \\
\hline 10 & 0.83 & 0.96 & 1.16 & - & - & - & - & - & - & 2.35 & 3.06 & 1.30 & 0.86 & 1.62 & 1.87 \\
\hline 30 & 0.93 & 1.32 & 1.42 & - & - & - & - & - & - & 3.17 & 2.84 & -1.11 & 0.80 & 1.24 & 1.55 \\
\hline 60 & 0.96 & 1.68 & 1.74 & - & - & - & - & - & - & 3.94 & 2.69 & -1.46 & 0.78 & 1.10 & 1.41 \\
\hline Brain (Avg) & & - & - & - & - & - & - & & & & & & & & \\
\hline 5 & 0.82 & 1.01 & 1.22 & - & - & - & - & - & - & 1.97 & 2.83 & 1.43 & 0.86 & 1.84 & 2.14 \\
\hline 10 & 0.87 & 1.03 & 1.19 & - & - & - & - & - & - & 2.31 & 3.17 & 1.37 & 0.88 & 1.79 & 2.03 \\
\hline 30 & 0.95 & 1.41 & 1.49 & - & - & - & - & - & - & 3.16 & 2.91 & -1.09 & 0.81 & 1.36 & 1.67 \\
\hline 60 & 0.97 & 1.84 & 1.91 & - & - & - & - & - & - & 3.99 & 2.72 & -1.47 & 0.79 & 1.22 & 1.54 \\
\hline
\end{tabular}

,$- \mathrm{n} / \mathrm{a}$.

volume of distribution than females for ketamine, and to a lesser extent NK, according to values obtained via noncompartmental analysis. It is therefore possible that this drug was more extensively distributed to other tissue and fluid compartments in male rats, which could help to explain its lower levels observed in plasma compared with females across the time period measured.

To this end, we examined ketamine and metabolite concentrations within the brain in two regions relevant to depressivelike behaviors and ketamine's related mechanism of action in rodents - the mPFC and HPC. Ketamine and NK were detected rapidly in the brains of male and female rats, both peaking 5-10 min after systemic administration of the parent compound. These findings agree with previous reports in mice and rats performed using similar routes of administration and confirm what is already known regarding distribution of ketamine after systemic and intravenous administration of the drug across species (Mion and Villevieille, 2013). As in plasma, ketamine levels were significantly higher in female versus male rats 5-10 min after injection. Norketamine peaked sooner in the brain than in plasma in both sexes, but was also detected at much greater levels in the mPFC and HPC of females compared with males over the first $90 \mathrm{~min}$ after administration. Unfortunately, DHNK levels detected were below the limit of quantification in both brain regions and were therefore not reported. Failure to detect reliably DHNK in the brain at a dose this low is unsurprising based on similar reports in rats administered low-dose ketamine systemically. Given that females displayed greater ketamine and NK concentrations than males both in the brain and plasma, greater distribution of ketamine to brain tissue in males cannot explain lower levels of ketamine and NK in males. Determination of drug levels in other tissues and compartments are needed to help explain the greater apparent volume of distribution observed in males.

To better understand the observed sex differences in ketamine concentrations over time, direct comparisons of pharmacokinetic parameters and concentration-time curves between the plasma and brain were made within each sex and then plotted against each other for further insight. First, to determine whether differences in metabolism of ketamine to downstream metabolites could help explain the greater female exposure to ketamine in the plasma and brain, cumulative ratios of total exposure levels (depicted by AUC values) for norketamine to ketamine were plotted over time at each measurement taken in the plasma and brain. Interestingly, despite greater concentrations of NK in the plasma of females compared with males, the NK:ketamine ratios were remarkably similar over the 180-min time period examined, with roughly five times greater norketamine exposure compared with ketamine in both sexes. This similarity was true at each time point examined, suggesting that metabolism of ketamine into NK does not contribute to differing plasma concentrations between male and female rats. In the brain, however, NK exposure was roughly twice that of ketamine in females, whereas males displayed an NK:ketamine ratio in equilibrium. Given that ketamine is not known to undergo local metabolism within the brain, this suggests that either distribution or permeability of NK into the brain is greater in females than in males, independent from the rate or extent of hepatic metabolism of the parent drug.

To examine this more, AUC values of ketamine and NK were compared between the brain and plasma in each sex and then plotted against each other for evaluation. In agreement 
with previous studies, ketamine levels were roughly three to four times greater in the brain than in plasma of both males and females; however, the rate of brain penetration of the parent compound differed between sexes. Over the first $10 \mathrm{~min}$ following ketamine administration, females displayed brain: plasma ketamine ratios of $\sim 3$ compared with $\sim 2$ in males. This trend shifted to favor males by $60 \mathrm{~min}$, who displayed nearly four times greater ketamine concentrations in the brain than in plasma, compared with $\sim 3$ times greater levels in females at this time point. Despite greater brain concentrations of ketamine in females, it is possible that males exhibit either slower elimination or greater retention of ketamine within the brain than females. Indeed, the mean residence time of ketamine was nearly $20 \%$ greater in males than in females. Differences in the rate of ketamine's elimination from the brain over time could have a significant impact on the time course of molecular consequences of ketamine action within the brain and, therefore, behavior at the low dose used in the present work.

Because NK is also known to be a pharmacologically active metabolite, sex differences in its concentrations within the brain also have the potential to influence ketamine's rapid behavioral effects. Owing to its reduced lipophilicity compared with ketamine, brain penetrance of NK was reduced relative to ketamine in both sexes. Norketamine brain:plasma ratios were near 1 in males, suggesting near equilibrium between the two compartments. This ratio was roughly doubled in females for the first 10 minutes following ketamine administration, but lowered to equilibrium by 60-90 minutes postdose. These data suggest similar elimination from and penetrance of NK in the brain, with slight differences in elimination rates for ketamine, between male and female rats, suggesting a low probability that these parameters contribute to the sex-dependent behavioral sensitivity to low-dose ketamine in rats. However, it is important to note that ketamine and NK displayed regional differences in concentrations in females, where concentrations of both were significantly greater in the mPFC than the HPC for the first 10 min after ketamine was administered. No difference was apparent in males, however, whose concentrations were similar in both regions examined. This sex discrepancy could help to explain the molecular differences between male and female rats shown to occur in both the $\mathrm{mPFC}$ and HPC rapidly following low-dose ketamine administration (Carrier and Kabbaj, 2013; Saland et al., 2016; Sarkar and Kabbaj, 2016). As this is the first report of regional differences in ketamine distribution within the brain between males and females, it is unknown the extent to which these differences occur throughout other regions of the brain that may have behaviorally relevant implications. It is an exciting finding, nonetheless, and warrants further investigation.

While the pharmacokinetic differences between male and female rats are certainly of interest, the lack of difference between proestrus and diestrus females are equally important to highlight when considering potential relevance of pharmacokinetic differences to those reported in depression-relevant behavioral assays. Despite differences in peripheral and central levels of estradiol and progesterone in proestrus and diestrus stages of the estrous cycle, levels of ketamine and NK in the plasma and brain tissue of these females were remarkably similar. Alone, this is not necessarily surprising, as no studies prior to the present work have investigated hormonal influence on pharmacokinetic parameters of ketamine in either rodents or humans. However, these findings hold great import when considered in the context of behavioral data reporting hormone-dependent effects of ketamine on depressive-like behavior in female rats. Whereas circulating estradiol and progesterone appear essential to the heightened behavioral sensitivity of female rats to low-dose ketamine, differences in their circulating levels do not appear to significantly affect metabolism or brain distribution of the same behaviorally relevant dose of ketamine in intact females (at least in the regions investigated herein). Therefore, it is more plausible that estrous cycle-dependent behavioral effects of a single low-dose of ketamine in rats are a consequence of pharmacodynamic, rather than pharmacokinetic, differences that occur with cyclic hormonal fluctuations. It should be noted that secondary metabolites, including HNK, were not examined in the present work. As such, estrous cycle-dependent differences in metabolism of NK to HNK and its distribution are unknown and warrant further investigation.

Given the single dose administration used in this study, these findings should not be generalized to repeated administration regimens. It is possible that estrous cycle could influence pharmacokinetic parameters of ketamine over repeated administration of a low dose. For example, estrous cycle appears to influence both maintenance of intravenous ketamine self-administration and reinstatement to ketaminepaired cues in rats (Wright et al., 2017). While pharmacokinetic differences between proestrus and diestrus rats are not apparent after a single systemic injection of low-dose ketamine, one cannot exclude the possibility that cycle-dependent reinforcing properties of ketamine following repeated administration (intravenous or otherwise) are influenced by pharmacokinetics. Future pharmacokinetic analyses of ketamine using different routes of administrations, doses, and treatment regimens (single vs. repeated) in females across the estrous cycle and males would greatly benefit comprehension of sex- and cycledependent behavioral and molecular differences reported across studies.

While this is the first investigation to examine and report pharmacokinetic sex differences of ketamine in brain and plasma of rats, conflicting findings recently were reported in mice (Zanos et al., 2016). Here, Zanos and colleagues found that higher HNK, but not ketamine or NK, levels are observed in the brain of female mice following acute administration of $10 \mathrm{mg} / \mathrm{kg}$ ketamine (intraperitoneally), in addition to greater female behavioral sensitivity to ketamine's antidepressantlike effects when compared with males. Additional experiments showed that systemically administered HNK is able to cross the blood-brain barrier and elicit antidepressant-like activity in mice without inducing ketamine-like side effects. However, sex differences were either not examined or not reported in this case, so it is unclear whether behavioral sensitivity to HNK differs between males and females. Here, it should be noted that females, but not males, exhibited an antidepressant-like response to $3 \mathrm{mg} / \mathrm{kg}$ ketamine, whereas both sexes responded to the $10 \mathrm{mg} / \mathrm{kg}$ dose used for pharmacokinetic analysis. Therefore, a direct association between greater HNK levels and enhanced female antidepressant-like response to ketamine cannot be inferred conclusively. In contrast, the present findings demonstrate greater ketamine and NK exposure in the plasma and brain of cycling female versus male rats following $2.5 \mathrm{mg} / \mathrm{kg}$ ketamine-a dose 
behaviorally effective in females but not males. In addition, the regional differences observed herein when the $\mathrm{mPFC}$ and hippocampus were examined independently may not be directly comparable to the whole brain analysis performed by Zanos et al. (2016). These findings further suggest species differences in not only behavioral, but also pharmacokinetic parameters following low-dose ketamine exposure, and highlight the need for pharmacokinetic analysis across multiple behaviorally relevant doses across species in both sexes.

Unfortunately, the lack of studies investigating sex differences in ketamine pharmacokinetics has left very little evidence regarding such effects in humans. The few that do exist suggest that observations of sex effects in ketamine metabolism and clearance are likely dose dependent. For example, $20 \%$ greater ketamine and NK clearance and lower drug/metabolite concentrations have been observed in healthy women compared with men following ketamine infusion at a higher dose $>10 \mathrm{mg} / \mathrm{kg}$ i.v. (Sigtermans et al., 2009). These sex differences were reflected at the behavioral level, with greater effects on cardiac output and heat pain-related indices in men than in women (Sigtermans et al., 2010). Conversely, Zarate and colleagues (2012) identified smaller sex differences in metabolism of low-dose ketamine in MDD and bipolar patients, where females displayed greater plasma levels of DHNK and HNK4a/c metabolites compared with males. Importantly, however, no sex differences in antidepressant response were apparent, and the differences in metabolite levels-notably that of HNK4a-had no significant association with treatment response. In fact, independent of sex, HNK5 was negatively associated with treatment response in bipolar depression patients (Zarate et al., 2012), suggesting that pharmacokinetic sex differences may not actually impact treatment response in clinical depression. Of note, hormone levels were not controlled for in these studies, which may have obscured potential differences in clinical response between sexes-particularly given the overlap in brain regions whose activity is modulated by both circulating ovarian hormone levels in women and ketamine itself (Arélin et al., 2015).

While underlying factors responsible for these varying differences in ketamine metabolism observed between males and females remain unknown, sex differences in hepatic expression and activity of ketamine-metabolizing cytochrome P450 enzymes are well-known (Waxman and Holloway, 2009) and subject to hormonal regulation by estrogen, progesterone, and testosterone, which also happen to be substrates of several P450 enzymes responsible for ketamine metabolism (Waxman and Holloway, 2009; de Vries and Forger, 2015). As well, physiologic differences influencing xenobiotic distribution, metabolism, and clearance (i.e., body weight, adipose tissue levels, and distribution) are present between males and females of a variety of species (de Vries and Forger, 2015). Ultimately, whether sex- and/or hormone-dependent pharmacokinetic processes contribute to differences between males and females in ketamine's antidepressant response is unclear, but the evidence strongly supports their consideration both preclinically and clinically. Likewise, non-negligible pharmacokinetic-related species differences have been highlighted herein, encouraging further examination to better translate findings between rodents and humans.

\section{Authorship Contributions}

Participated in research design: Saland, Kabbaj.

Conducted experiments: Saland.

Performed data analysis: Saland.

Wrote or contributed to the writing of the manuscript: Saland, Kabbaj.

\section{References}

Abdallah CG, Adams TG, Kelmendi B, Esterlis I, Sanacora G, and Krystal JH (2016) Ketamine's mechanism of action: a path to rapid-acting antidepressants. Depress Anxiety 33:689-697.

Arélin K, Mueller K, Barth C, Rekkas PV, Kratzsch J, Burmann I, Villringer A, and Sacher J (2015) Progesterone mediates brain functional connectivity changes during the menstrual cycle-a pilot resting state MRI study. Front Neurosci 9:44.

Becker JB, Arnold AP, Berkley KJ, Blaustein JD, Eckel LA, Hampson E, Herman JP, Marts S, Sadee W, Steiner M, et al. (2005) Strategies and methods for research on sex differences in brain and behavior. Endocrinology 146:1650-1673.

Becker JB, Prendergast BJ, and Liang JW (2016) Female rats are not more variable than male rats: a meta-analysis of neuroscience studies. Biol Sex Differ 7:34.

Carrier N and Kabbaj M (2013) Sex differences in the antidepressant-like effects of ketamine. Neuropharmacology 70:27-34.

de Vries GJ and Forger NG (2015) Sex differences in the brain: a whole body perspective. Biol Sex Differ 6:15.

Hollis F, Duclot F, Gunjan A, and Kabbaj M (2011) Individual differences in the effect of social defeat on anhedonia and histone acetylation in the rat hippocampus. Horm Behav 59:331-337.

Keers R and Aitchison KJ (2010) Gender differences in antidepressant drug response. Int Rev Psychiatry 22:485-500.

Mion G and Villevieille T (2013) Ketamine pharmacology: an update (pharmacodynamics and molecular aspects, recent findings). CNS Neurosci Ther 19:370-380.

Patel V, Vikram P, Dan C, Rachana P, Charlson FJ, Louisa D, Tarun D, Ferrari AJ, Steve H, Ramanan L, et al. (2016) Addressing the burden of mental, neurological, and substance use disorders: key messages from Disease Control Priorities, 3rd edition. Lancet 387:1672-1685.

Saland SK, Duclot F, and Kabbaj M (2017) Integrative analysis of sex differences in the rapid antidepressant effects of ketamine in preclinical models for in dividualized clinical outcomes. Curr Opin Behav Sci 14:19-26.

Saland SK, Schoepfer KJ, and Kabbaj M (2016) Hedonic sensitivity to low-dose ketamine is modulated by gonadal hormones in a sex-dependent manner. Sci Rep 6:21322.

Sarkar A and Kabbaj M (2016) Sex differences in effects of ketamine on behavior, spine density, and synaptic proteins in socially isolated rats. Biol Psychiatry 80: 448-456.

Sigtermans M, Dahan A, Mooren R, Bauer M, Kest B, Sarton E, and Olofsen E (2009) $\mathrm{S}(+)$-ketamine effect on experimental pain and cardiac output: a population pharmacokinetic-pharmacodynamic modeling study in healthy volunteers. Anesthesiology 111:892-903.

Sigtermans M, Noppers I, Sarton E, Bauer M, Mooren R, Olofsen E, and Dahan A (2010) An observational study on the effect of S+-ketamine on chronic pain versus experimental acute pain in Complex Regional Pain Syndrome type 1 patients. Eur J Pain 14:302-307.

Waxman DJ and Holloway MG (2009) Sex differences in the expression of hepatic drug metabolizing enzymes. Mol Pharmacol 76:215-228.

Wright KN, Strong CE, Addonizio MN, Brownstein NC, and Kabbaj M (2017) Reinforcing properties of an intermittent, low dose of ketamine in rats: effects of sex and cycle. Psychopharmacology (Berl.) 234:393-401.

Zanos P, Moaddel R, Morris PJ, Georgiou P, Fischell J, Elmer GI, Alkondon M, Yuan P, Pribut HJ, Singh NS, et al. (2016) NMDAR inhibition-independent antidepressant actions of ketamine metabolites. Nature 533:481-486.

Zarate CA Jr, Brutsche N, Laje G, Luckenbaugh DA, Venkata SLV, Ramamoorthy A, Moaddel R, and Wainer IW (2012) Relationship of ketamine's plasma metabolites with response, diagnosis, and side effects in major depression. Biol Psychiatry 72: 331-338.

Zarate CA Jr, Mathews DC, and Furey ML (2013) Human biomarkers of rapid antidepressant effects. Biol Psychiatry 73:1142-1155.

Zhang Y, Huo M, Zhou J, and Xie S (2010) PKSolver: An add-in program for pharmacokinetic and pharmacodynamic data analysis in Microsoft Excel. Comput Methods Programs Biomed 99:306-314.

Address correspondence to: Mohamed Kabbaj, College of Medicine, Florida State University, 1115 W Call Street, Tallahassee, FL 32306. E-mail: mohamed.kabbaj@med.fsu.edu 